\title{
FORMACIÓN UNIVERSITARIA EN SALUD: UNA MIRADA DESDE LAS PRÁCTICAS PEDAGÓGICAS
}

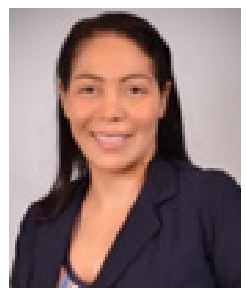

\section{Arley Denisse Vega Ochoa}

Universidad Metropolitana de Educación, Ciencia y Tecnología, Panamá arleyvega@unicesar.edu.co
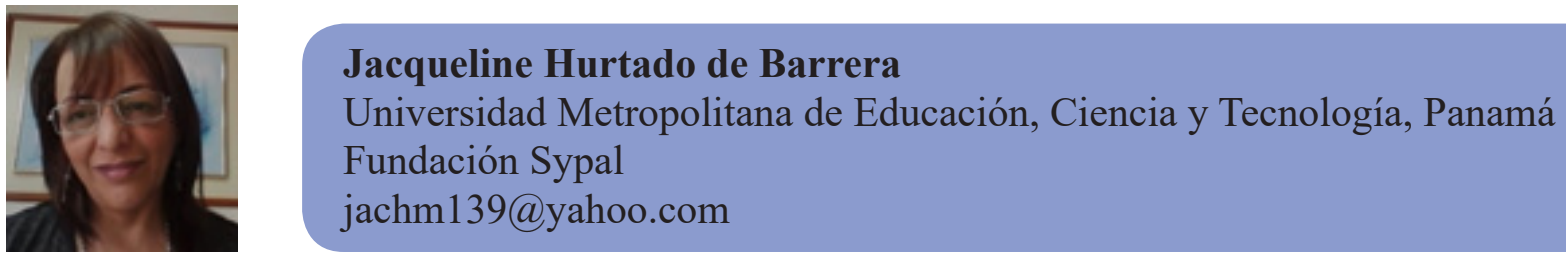

\section{RESUMEN}

En el presente ensayo se realiza una reflexión sobre la formación del docente universitario del área de la salud con énfasis en las prácticas pedagógicas, como una necesidad de compromiso de las universidades que realizan la formación de un profesional competente en las dimensiones del ser, saber, saber hacer y saber convivir, que permita al futuro profesional insertarse en el sector productivo con capacidad para resolver problemáticas a lo largo de la vida. La problemática de la educación superior en Colombia sigue siendo uno de los principales temas de discusión en los diferentes espacios académicos de este país, de manera que se sigue pensando en los métodos y prácticas de forma disciplinar, a la hora de enseñar, por tanto, se requiere repensar desde una perspectiva crítica las prácticas pedagógicas en los programas académicos que ofertan carreras de salud con el propósito de viabilizar algunas transformaciones en el proceso de enseñanza y desde una concepción transdisciplinaria. En el caso de los docentes del área de la salud, aunque tienen dominio de su área disciplinar, presentan dificultades para orientar la enseñanza basada en competencias, lo que requiere que el docente desarrolle las habilidades orientadas a incorporar y complementar prácticas pedagógicas bajo un sistema de estrategias educativas, que no solo se orienten a impartir conocimientos en un área específica, sino que además fomenten el proceso de enseñanza y aprendizaje significativo.

Palabras clave: Formación universitaria; formación en salud; competencias en salud; prácticas pedagógicas. 


\title{
UNIVERSITY TRAINING IN HEALTH: A LOOK FROM PEDAGOGICAL PRACTICES
}

\begin{abstract}
In the present essay makes a reflection about the teacher training of the university in the health area with emphasis on pedagogical practices, as a need for commitment of the universities that carry out the training of a competent professional in the dimensions of being, knowledge, know how to do and know how to coexist, that allows the future professional to be inserted in the productive sector with capacity to solve problems throughout life. The problem of higher education in Colombia continues to be one of the main topics of discussion in the different academic spaces of this country, so that we continue to think about methods and practices in a disciplinary way, when teaching, therefore, it is necessary to rethink from a critical perspective the pedagogical practices in the academic programs that offer health careers with the purpose of making possible some transformations in the teaching process and from a transdisciplinary conception. In the case of teachers in the health area, although they have mastery of their disciplinary area, they have difficulties to guide competency-based teaching, which requires the teacher to develop the skills aimed at incorporating and complementing pedagogical practices under a system of educational strategies, which not only aim to impart knowledge in a specific area, but also encourage the process of significant teaching and learning.
\end{abstract}

Keywords: University education; health training; health competencies; pedagogical practices.

\section{INTRODUCCIÓN}

Cada vez más, los avances de la ciencia y los conocimientos aumentan aceleradamente, y exigen que el sistema educativo ofrezca una educación contextualizada, y responda en consonancia con currículos y pedagogías pertinentes.

Las constantes transformaciones de la educación en el mundo globalizado, requieren que las instituciones de educación superior tengan a su disposición, personal altamente competente para llevar a cabo las funciones sustantivas de las universidades como son la docencia, la investigación y la extensión, para favorecer los procesos de calidad. La educación encaminada a la transformación personal y colectiva, se ha planteado más como proceso, en tanto que determina unos ideales específicos de sociedad, que apuntan a transformar el orden político, económico, social y cultural.

En este proceso de transformación de la educación se desarrolla el aprendizaje del estudiante en formación, que parte inicialmente de la acumulación de teorías que se asocian en la práctica 
mediante la resolución de problemáticas del contexto que contribuyen significativamente al proceso de aprendizaje. El aprendizaje es la construcción y adquisición de conocimiento, competencias, habilidades, prácticas y cualidades, por medio del estudio y de la experiencia.

En efecto el aprendizaje no sólo es la adquisición de los procesos cognitivos, va más allá de la capacidad de utilizar esos conocimientos para enfrentar problemáticas que se presentan a lo largo de la vida. Esto ratifica que el docente debe comprometerse con el proceso de enseñanza, ya que se convierte en un actor fundamental en la formación del futuro profesional. El proceso de enseñanza, es parte esencial de la educación. En la reinvención de la pedagogía se le ha dado gran importancia al proceso de enseñanza como una mediación de procesos significativos que se construyen (Díaz Barriga, 2006).

La enseñanza consiste en planear, organizar y preparar las condiciones para que las personas aprendan. Estas condiciones van desde el discurso coherente, argumentado del docente, hasta el desarrollo de distintas experiencias de los estudiantes, quienes, dirigidos por un orientador, exploran y se introducen en el mundo de la cultura, la ciencia, el arte, la tecnología y la sociedad. Por lo tanto, los docentes deben examinar constantemente las técnicas, los procedimientos y los principios que rigen su intervención como docentes, puesto que son los responsables de imprimir motivación, hábitos de aprendizaje y autorregulación en sus estudiantes.

\section{LA FORMACIÓN UNIVERSITARIA EN EL ÁREA DE LA SALUD}

La formación de los profesionales del área de la salud requiere de una exigencia para los profesores porque su misión es formar personas competentes con un amplio grado de responsabilidad debido a que el objeto de estudio de estas carreras se basa en el cuidado de la vida y la salud.

Para 2019 existen 15 profesiones del área de la salud reconocidas en el Colombia, certificadas por el Ministerio de Educación Nacional y el Ministerio de Salud y Protección Social, de acuerdo a lo establecido en la ley 1164 de 2007 para el ejercicio de las profesiones del área de la salud, (Men, 2009). Las carreras profesionales en salud, especialmente Medicina, cuentan de una alta demanda en el ámbito universitario. Sin embargo, la expectativa de inserción en el mercado laboral es poco viable por el alto nivel de desempleo, además la salud en Colombia pasa por una situación crítica, debido a que existe cierta inestabilidad laboral y los ingresos salariales son muy bajos y poco oportunos.

En relación con lo referido, según el SNIES (2017), solo en el año 2017 y en la carrera de medicina, sin contar, odontología, instrumentación, biología, cirugía, pediatría, y enfermería que 
son carreras asociadas al sistema de salud, se presentaron 2907 alumnos inscritos en instituciones de educación superior, lo cual reafirma la existencia de los altos índices académicos para el ingreso de estudiantes preuniversitarios en el área de medicina.

Cuadro $\mathbf{N}^{\circ}$ 1. Relación de inscritos en programas del área de la salud en Colombia, año 2017

\begin{tabular}{|l|c|}
\hline \multicolumn{1}{|c|}{ Carreras universitarias en Colombia } & Total, inscritos año 2017 \\
\hline Medicina & 87.664 \\
\hline Enfermería & 28.861 \\
\hline Instrumentación Quirúrgica & 1.682 \\
\hline
\end{tabular}

Fuente: (SNIES, 2017).

Para el profesional del área de la salud su principal función está centrada en el cuidado de la vida a través de la promoción, la prevención, el tratamiento y/o la rehabilitación de una patología que afecta a la persona que busca mejorar su calidad de vida. Hoy en día la medicina se ha quedado en una visión reduccionista de la enfermedad, y en consecuencia se estudia sólo el miembro enfermo como una parte, sin tener en cuenta todo el sistema, lo que permite ver la enfermedad desde un punto de vista, y no desde su complejidad.

La enfermedad ha sido reducida a mecanismos biológicos, estudiados bajo la óptica de la biología celular y molecular, y se han evacuado completamente los aspectos psicológicos y sociales de la enfermedad. Aunque el conocimiento de los aspectos fisiológicos sea, por supuesto, útil, el enfoque reduccionista no suministra más que una visión de las cosas (Duque, Rodríguez y Vallejo, 2013).

Así mismo, el sistema de educación profesional en salud está orientado a la organización de contenidos ya elaborados, agrupados en estructuras curriculares por asignaturas, lo que hace que el conocimiento sea fragmentado, y que el profesional en formación reciba información teórica para luego aplicarla en la práctica. Los contenidos se presentan aislados, y no desde la transdisciplinariedad, lo que hace que el estudiante piense que las asignaturas del ciclo básico no le sirven de nada para su formación profesional.

Esa fragmentación se refleja en el sistema de salud, ya que la enfermedad se maneja como una parte, sólo se estudia el órgano afectado y no a la persona en su sistema total. En el área de la salud un médico no puede recetar la misma medicina a todos los pacientes, lo mismo pasa con los profesionales en formación, los estudiantes no tienen el mismo estilo de aprender, es por eso que el docente no debe enseñar de la misma manera a todos y en todas las asignaturas, por lo tanto, se 
hace necesario que cada día incorpore transformaciones a su forma de enseñar.

De la necesidad de profesores preparados para trabajar en el área de salud, nace la relevancia de preparar docentes profesionales que posean profundo conocimiento, no solo en el área médica, sino que además cuenten con las herramientas pedagógicas pertinentes que vayan en pro de una mejora continua en el sistema educativo, para que los jóvenes de hoy en día respondan a las exigencias mundiales de la globalización, tanto culturales, económicas y educativas, lo que requiere realizar cambios para generar un mayor índice de exigencia y a su vez mayor calidad humana en la incursión de los procesos de enseñanza.

Cabe destacar, en referencia a las competencias del docente en el área de formación en salud, que éstos deben fomentar el trato humanizado y la sensibilización en los estudiantes desde las primeras etapas de su educación (Díaz et al 2018). Además, el docente debe contar con la didáctica necesaria para incluir la investigación en el área de salud, pues, estos docentes tienen mayor responsabilidad en los procesos de indagación acerca de la solución de problemas sociales y educativos (Perrenoud 2004).

En este sentido se hace evidente la necesidad de incorporar las competencias en el aula, para pasar de estructuras lineales y fragmentadas con recortes de contenido, y de una educación poco práctica que no dota de recursos para adaptarse a una sociedad cambiante, a modelos curriculares interdisciplinarios que permitan contextualizar el conocimiento a través de un saber actuado, de manera que el estudiante pueda resolver problemas de salud de la comunidad.

Las facultades de salud y sus programas académicos, reconocen la necesidad de asumir la transformación de los estudiantes para un mundo competitivo y sostenible, con un profesional capaz de resolver problemáticas que se presentan a lo largo de la vida. En la misma línea los programas académicos apuntan al logro de los requerimientos que exige el Ministerio de educación nacional de reconocer a la sociedad las condiciones de calidad con las que cumplen las Instituciones de educación superior al formar profesionales competentes al servicio de la comunidad.

Para Duque, Rodríguez, y Vallejo (2013), la atención en salud requiere que los profesionales desarrollen destrezas en el reconocimiento de problemas; en la recolección de datos, en la organización del pensamiento y habilidades en la toma de decisiones, en la relación con el paciente y la comunidad. Esto implica que el profesional docente del área de la salud además de conocer su disciplina debe poseer competencias metodológicas para orientar el proceso de enseñanza. Esta debilidad se ve en los profesionales de la salud que se dedican a la enseñanza hoy en día, pues 
conocen mucho su área disciplinar, pero tienen falencias al momento de enseñar.

Entonces, lo anterior lleva a los docentes a preguntarse, ¿ser un buen profesional es directamente proporcional a ser un buen docente? La respuesta es no; ser un buen profesional implica tener un conocimiento pleno, académico y práctico, complementado por la ética y la moral en las acciones desempeñadas en su área académica. No obstante, ser buen docente requiere de una serie de cualidades más profundas que no sólo dejan ver las estrategias pedagógicas didácticas, y los procesos meta cognitivos en el educador, sino que dejan ver mucho más allá del área profesional educativa. Ser un buen profesional y docente a la vez implica interrelacionar aspectos académicos, metodológicos, investigativos, sociales, personales y de calidad humana sobre todo en el área de la salud.

No es suficiente ser un excelente profesional, a la hora de enseñar es muy importante saber utilizar las técnicas y prácticas adecuadas para que el estudiante aprenda, se requiere crear y recrear un entorno estimulante, para que se desarrolle el aprendizaje autorregulado. Ser profesor, como ser profesional, requiere de competencias propias y diversificadas: se puede ser bueno en una de ellas y mediocre en la otra (Zabalza, 2016). Cada condición tiene sus competencias y exigencias específicas. Desde esta perspectiva Parra y Galindo (2016), destacan que el conocer una disciplina no hace maestros, éstos se forman y reconstruyen a sí mismos en el campo de acción.

Otra situación que se presenta comúnmente en los docentes del área de salud, es la aplicación de estrategias según sus conocimientos y sus vivencias a lo largo de su trabajo educativo y profesional previo, situación que en ocasiones conlleva al docente a impartir conocimientos según su experiencia, y no con base en teorías educativas, ni en y conocimiento actualizado, por lo tanto, usa pocas estrategias didácticas, y esta situación afecta al estudiante.

En la misma línea, los docentes suelen orientar su práctica pedagógica de la manera como ellos aprendieron, algunos imitan a sus propios docentes, otros son orientados por sus colegas, y muy pocos hacen cursos extracurriculares. Como los docentes fueron formados en un pensamiento pasivo, coartado, y centrado en repetir esquemas de semestre a semestre, siguen repitiendo estas rutinas generación tras generación, sin considerar que los tiempos cambian y que el conocimiento se transforma constantemente. A esta realidad se suma que quienes se desempeñan como docentes en el área de la salud realizan labores empíricas de enseñanza, y se basan en construcciones propias, en su experiencia o en modelos aprendidos (Ángel y Rojas, 2017).

A menudo, ingresan profesionales al campo de la educación superior sin tener formación 
docente y complementaria para trabajar como profesores, que terminan repitiendo la misma manera convencional de enseñanza en la que fueron formados, sin preparación para cumplir con las nuevas necesidades profesionales requeridas. A la falta de formación didáctica, se suma la falta de preparación profesional, que en ocasiones es consecuencia de la formación académica recibida en la carrera y del tipo de educación adquirida en los estudios de posgrado.

Otro aspecto preocupante tiene que ver con el dominio que los docentes tienen de las Tic. En un estudio realizado por Zempoalteca, Barragán, González y Guzmán (2017), en instituciones públicas de educación superior, se aplicó un instrumento de recolección de datos para determinar las competencias docentes en el uso de las Tic, el acceso y disponibilidad de Tic en la institución, y la incorporación de las Tic en actividades docentes. Los resultados evidenciaron la falta de preparación de los docentes reflejada dificultades para solucionar problemas, para construir, identificar y acrecentar su propia identidad cultural, y ser partícipes activos y críticos de las soluciones que el país demanda.

Dentro del quehacer docente en el campo de la salud, es también muy importante comprender la relación entre hábitos de estudio y acompañamiento pedagógico. Esto necesariamente remite asumir una teoría de la educación centrada en la persona, por cuanto se busca, hacer aproximaciones contextuales con relación al componente humano que se da en la relación entre quien orienta el proceso de enseñanza y quien aprende. El acto educativo debe estar apoyado por las actitudes y los hábitos de estudio que se fortalecen desde los procesos educativos, y que van caracterizando el autoaprendizaje y el aprendizaje autónomo del estudiante.

Un camino para favorecer la calidad académica en el campo de la salud, es definir, no sólo lo que se quiere enseñar, sino cómo se va a hacer y los recursos a utilizar, para facilitar un proceso de enseñanza y de aprendizaje eficiente. No se trata de decidir "que" va a enseñar el docente sino el "como" va a enseñar el contenido, el método y los recursos, para lo cual requiere una planificación del proceso de enseñanza acorde con el contexto, con el tipo de contenido y con las competencias deseadas.

De acuerdo a lo expuesto, se hace referencia a lo que significa para Tobón (2013), ser un buen mediador educativo en el proceso de aprendizaje de los jóvenes en el área de salud:

- Explorar las potencialidades que posee el estudiante en las diferentes áreas de desarrollo (cognitiva, actitudinal y procedimental).

- Determinar las necesidades de aprendizaje.

- Negociar el aprendizaje significativo que ha de obtenerse mediante actividades que 
interesen a los estudiantes y generen la necesidad de aprender.

- Ofrecer ayuda a partir de las dificultades manifiestas. No adelantarse ni dar por supuestas determinadas necesidades de los estudiantes.

- Dar libertad, de manera responsable y comprometida, para hacer y crear. Propiciar la autorregulación individual y grupal, y la autogestión del equipo.

- Enseñar a procesar y sistematizar la información.

- Permitir el error y la autorregulación, para aprender de la respuesta incorrecta.

- Respetar estilos y ritmos de aprendizaje.

- Precisar el resultado de aprendizaje esperado.

- Propiciar la expresión por diversas vías.

Por otra parte, entre las dificultades que generalmente presenta el docente como mediador en el sistema educativo, están el no participar del sistema total, el generar dependencia en el estudiante y el no lograr el desarrollo de sus propias competencias. Esto es preocupante, en especial porque el área de salud requiere mayor participación docente, sobre todo en los procesos prácticos de enseñanza, y también amerita un autodesarrollo y autogestión de los estudiantes en la administración del conocimiento.

A esta situación se añade la problemática de los docentes en cuanto a la actualización de competencias docentes, tanto prácticas como teóricas, donde juegan un papel importante ciertos factores que generan resistencia en el docente para su participación en la generación de nuevas propuestas curriculares. Según Medina y Santeliz, (2008), dichos factores hacen referencia a:

- El tiempo de contratación a medio tiempo y tiempo ocasional y catedrático.

- Elevada carga académica.

- Compromisos adquiridos en diversas comisiones de trabajo en la universidad.

- Poca promoción del proyecto por parte de las autoridades.

- Mayor importancia al aspecto asistencial en los docentes del área clínica.

- Escasa o ninguna capacitación en el área de las competencias

- Temor al cambio

- El nuevo diseño implica una manera diferente de hacer las cosas

- Incertidumbre en relación con los alcances académicos de la propuesta, bases teóricas y proceso de evaluación de las competencias

- Percepción de una imposición desde la alta gerencia

- Indiferencia o desinterés en el proyecto

A partir de lo expuesto, se presentan aspectos que realmente representan dificultad en los 
docentes para fortalecer las competencias y fomentar el currículo del perfil del profesional en educación. A continuación, se presenta una estadística de las evaluaciones en referencia a la actitud de los docentes con respecto al perfil profesional basado en el enfoque de competencias.

Gráfico $\mathbf{N}^{\circ}$ 1. Actitud de los docentes hacia el diseño curricular basado en competencias.

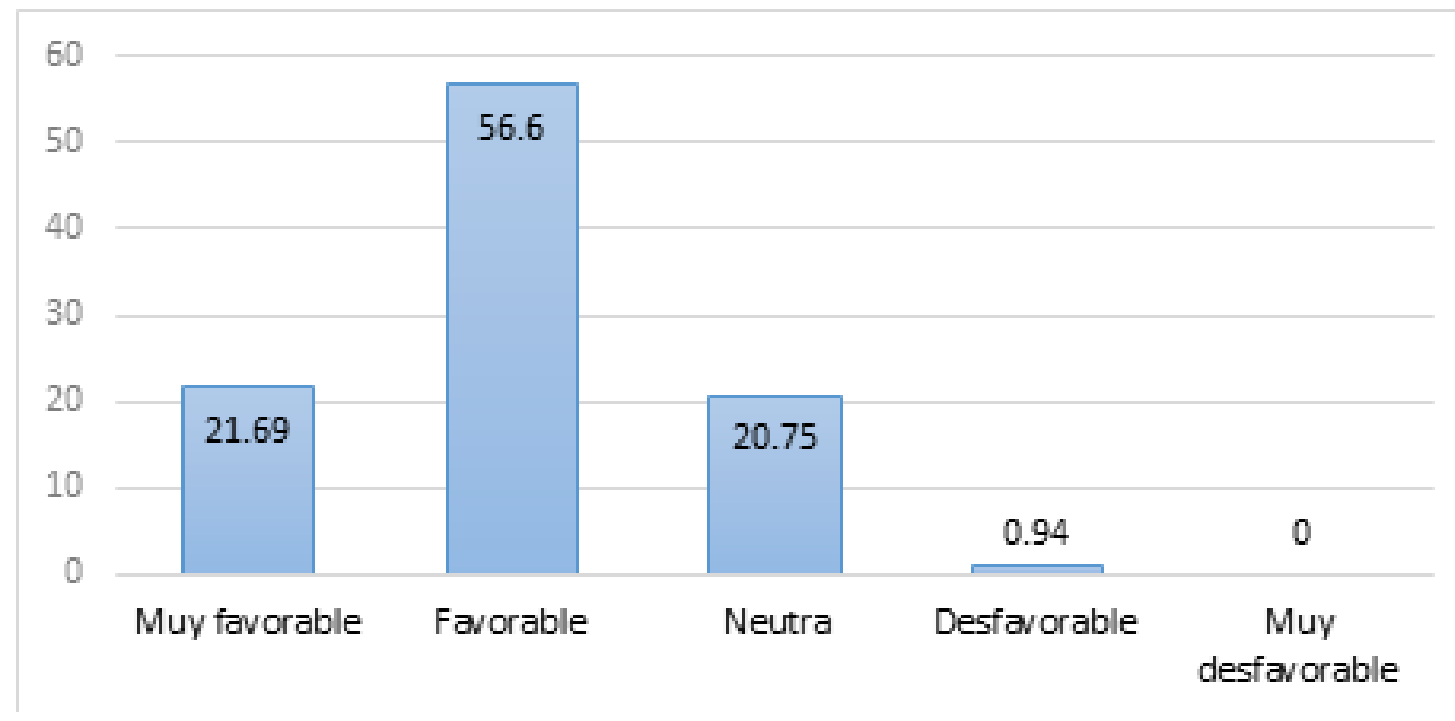

Fuente: (Medina y Santeliz, 2008)

De acuerdo a Medina y Santeliz (2008), los resultados expuestos, muestran que el $21.69 \%$ de los profesores tuvo una actitud muy favorable con respecto a los cambios al perfil docente, el 56.6 $\%$ tuvo una actitud favorable, el 20.75 neutra y $0,94 \%$ desfavorable. Ninguno expresó una actitud muy desfavorable. En adelante correspondería a la institución hacer una gestión eficaz y eficiente en pro de fomentar un desarrollo curricular orientado a las competencias docentes en el área de salud.

A partir de los resultados emitidos en investigación de Medina y Santeliz (2008), cabe destacar la relevancia del cuerpo directivo de las universidades en el fomento de las competencias docentes en el área de salud, por ello, se hace imprescindible trabajar en la descentralización del área docente y en los factores motivadores del perfil curricular docente.

En este sentido, Calderón (2012), presenta un análisis histórico de los procesos de descentralización en la gestión educativa superior, y plantea que las instituciones educativas deben enfrentarse al gobierno para lograr ejecutar un proceso educativo independiente y relacionado con las mejoras continuas en la calidad, tanto de gestión administrativa, como en los procesos de gestión docente en el área de salud. 
Calderón (2012), refiere que es necesario hablar de una actualización en el perfil del docente en salud, y para lograrla, la gestión educativa en el área gerencial juega un papel importante en las universidades, pero en ocasiones esto se complica debido a la centralización educativa de los gobiernos, que intervienen, a veces de forma poco idónea, con una cantidad de restricciones que limitan la democracia en los sistemas educativos por competencias, a su vez esto podría generar consecuencias negativas en la educación de los estudiantes en formación en el área de salud.

En la misma línea, la gestión educativa no solo depende del proceso de promover el perfil curricular del docente en el área de salud, además depende de muchos aspectos. En todo caso, un adecuado proceso educativo debe contemplar procesos eficaces tanto teóricos como prácticos, para fomentar el desarrollo de los estudiantes en el contexto real, y poner en práctica sus conocimientos.

Las prácticas pedagógicas deben ser un medio que permita al estudiante desarrollar el pensamiento crítico, creativo e innovador. Se requiere utilizar nuevos métodos que vayan más allá del dominio de la disciplina y aplicar nuevos planteamientos pedagógicos y didácticos, por lo cual el docente debe adoptar el rol mediador y orientador para que el estudiante logre un aprendizaje significativo, y de esta manera se facilite intencionalmente un procesamiento más profundo de la información nueva planeada por el docente. También es importante el desarrollo de diversas estrategias a través de propósitos del aprendizaje, como son los resúmenes, ilustraciones, organizadores previos, preguntas intercaladas, pistas tipográficas y discursivas, analogías, mapas conceptuales y redes semánticas, uso de estructuras textuales a través del aprendizaje basado en resolución de problemas, aprendizaje colaborativo; de tal manera que se induzca al estudiante a desarrollar y fortalecer su capacidad cognitiva.

La práctica pedagógica del docente universitario se asume como un proceso multidimensional en el que se expresan representaciones, cogniciones, significados y expectativas de los actores que participan, y se asumen roles en situaciones de interdependencia (Zúñiga y Jopia, 2007). En opinión de Zabalza (2009), la práctica pedagógica incorpora una serie de dimensiones como la planificación, la selección de contenidos, la contextualización de la enseñanza, el manejo de tecnologías y la organización de actividades y la evaluación del aprendizaje.

Cada uno de los elementos se relaciona entre sí para el desarrollo de una buena práctica pedagógica acorde con las necesidades del estudiante y el perfil de egreso del plan de estudios de cada carrera. El docente, desde el deber ser, y como orientador de los procesos pedagógicos, se consolida como una figura mediadora, con un profundo conocimiento disciplinar, teórico, practico, 
tecnológico e investigativo, además de conocimiento didáctico y ético, con el propósito de asumir la relación entre el discurso que promueven las instituciones educativas y las acciones que debe realizar dentro del aula (Basto, 2011).

\section{LA NECESIDAD DE PROFESORES PREPARADOS EN DIDÁCTICA}

La generación y el uso de un conocimiento innovador y crítico, involucra factores de enseñanza que promueven un aprendizaje autónomo, de autogestión del conocimiento, y activa el aprendizaje, para favorecer la construcción de un saber que engloba toda la dimensión de la persona en el acto de conocer, producir conceptos y solucionar problemas.

Igualmente, la formación basada en competencias se debe enmarcar dentro del currículo profesional del docente en el área de salud. Las habilidades y destrezas propias de esta área, requieren prácticas de simulación para vivir experiencias que se puedan aplicar a la práctica real. De acuerdo a lo anterior el estudiante adquiere competencias para asumir las situaciones reales que se presentan en el día a día en su vida del profesional.

Los cuatro saberes de los que el estudiante debe apropiarse son: el saber teórico o científico, el saber práctico, el saber hacer o praxis, el saber convivir, y el saber ser como producto final, que consiste en la reflexión, seguida de una acción fundada en razones analíticas con capacidad de valorar al otro. La educación centrada en la persona implica poner el foco en el aprendizaje, y hacer del estudiante, gestor principal y partícipe activo de su propio aprendizaje, aspecto que ha sido abordado por diferentes organismos y autores, quienes desde diversas posiciones y perspectivas proponen definiciones al respecto.

En educación, la tarea es conseguir que los estudiantes aprendan, aunque su resultado no siempre responda a las metas y expectativas consensuadas en el acto educativo, ni a los esfuerzos metodológicos.

Crispín y otros (2011), reconocen que en el aprendizaje influyen diversos factores como la capacidad, la motivación, los conocimientos previos y las estrategias de aprendizaje, que son el conjunto de actividades, técnicas y medios que se planifican de acuerdo con las necesidades de los estudiantes, los objetivos que se buscan y la naturaleza de los conocimientos, con la finalidad de hacer efectivo el proceso de aprendizaje.

Para Zahonero y Bris (2012), la calidad de la educación superior es una condición básica para realizar un correcto trabajo en la formación del recurso humano, pero también en el desarrollo 
de nuevos conocimientos concebidos como un servicio a la sociedad. Y aunque es evidente que el concepto de calidad educativa está sujeto a controversias, por encima de todo prevalece la convicción de que la búsqueda de la calidad en educación debe aspirar a que la universidad sea una comunidad de aprendizaje permanente, como condición indispensable.

Para Zahonero y Bris (2012), en el perfil característico de un buen profesor, se destacan las competencias que acentúan el dominio de la materia junto a la preocupación por mantener la metodología y los contenidos al día, además de la capacidad de indagación e investigación continua, la habilidad para diseñar, planificar y gestionar el currículum en colaboración con otros docentes dentro de una disposición favorable al necesario trabajo en equipo.

Para el aprendizaje de las carreras del área de salud es necesario desarrollar la práctica en forma paralela con los contenidos teóricos para que el estudiante pueda contextualizar lo aprendido, lo que permite el desarrollo de la competencia en el ejercicio de la formación. En efecto esta situación hace que el docente sea un eje fundamental en el proceso de enseñanza como orientador y guía, lo que exige el dominio de sus competencias didácticas para asumir los retos de una práctica pedagógica conforme a las necesidades de la globalización.

Para Henao (2010), identificar, valorar y desarrollar las competencias constituye todo un desafío para enfrentar las nuevas tendencias educacionales que implican un cambio en la conducción de los procesos educativos. Acosta (2015), considera que las competencias son un conjunto de conocimientos, habilidades, destrezas, actitudes, aptitudes y valores que toman parte activa en el desempeño responsable y eficaz de un individuo para el ejercicio de un conjunto de actividades cotidianas dentro de un contexto determinado.

Según Tobón, (2013), la educación debe contextualizar el saber en lo local, lo regional y lo internacional, que permita a los estudiantes, docentes y administrativos ir más allá de la simple asimilación de conocimientos y pasar a una dinámica de búsqueda, selección, comprensión, sistematización, critica, creación, aplicación y transferencia.

La llamada formación por competencias se viene aplicando desde la década de los noventa en las instituciones de educación superior, con el propósito de formar personas competitivas en el contexto laboral. De ahí que se hace necesario realizar cambios profundos en el proceso de enseñanza que implica compromiso del docente en la formación de calidad. La realidad de hoy es que generalmente el docente toma una postura tradicional donde él es el transmisor de conocimientos y el estudiante se limita a escuchar lo que dificulta la construcción del conocimiento 
a partir del planteamiento del profesor.

Cuando se da un mayor énfasis a la acción entre el educador y el saber, se logra tener una forma de instrucción estricta, ligada a las pedagogías más tradicionales, pues lo que se desarrolla es el ámbito de la enseñanza, más que el de aprender a ser reflexivo y cuidadoso con la labor de aprendizaje.

\section{CONCLUSIONES}

En primera instancia se menciona que la problemática genera una incertidumbre en los profesionales en formación por su futuro laboral, pero a pesar de todo, las personas se vinculan a carreras del área de la salud y posteriormente a programas de posgrado con la finalidad de hallar un futuro deseable para tener mejores oportunidades de empleo.

La prueba Saber Pro no es un examen de memoria. Más bien se contiene ítems en gestión y resolución de problemas de carácter general y disciplinar, lo que hace que la prueba sea de un nivel de complejidad ascendente. Esta situación hace que el docente deba repensar el modelo de enseñanza para que promueva en la práctica pedagógica el pensamiento complejo, y la relación entre la interdisciplinariedad y el saber disciplinario.

La principal misión de los profesionales de la salud es el cuidado de la vida y la salud de las personas independientemente de su condición, con la finalidad de preservar la vida y seguridad de la población que requiere de sus cuidados. Consecuente con esta situación las universidades y los docentes realizan esfuerzos intelectuales en la comunidad académica, que propenden a optimizar los procesos pedagógicos para mejorar la capacidad de aprender a aprehender y fomentar el pensamiento resolutivo, crítico e innovador, y dar respuesta a una formación disciplinar integrada.

Además de todo, para ingresar al sector productivo, los egresados deben participar en el modelo de evaluación por competencias estandarizado a nivel internacional, ya que en Colombia las empresas evalúan con el modelo Saber Pro con el que evalúan las universidades.

Como complemento, cabe destacar, la relevancia de la gestión educativa independiente en las universidades. Dicha gestión debe enfatizar la preparación y actualización de conocimientos en el personal docente. De igual forma, los docentes y las universidades deben fomentar tanto la actualización de la pensa académica en salud, como la promoción de las estrategias pedagógicas y didácticas en el área de salud, que a la final deben sustentar la practica pedagógica profesional en el contexto real de situaciones en los laboratorios de simulación. 
Bajo este orden de ideas, es importante delimitar en concreto, las pautas primordiales que el docente debe poseer, y fomentar en el proceso de aprendizaje, Además debe potenciar, en los futuros egresados, el sentido de pertenencia al área de salud, el empleo de los procesos éticos, la humanización y sensibilización en su área laboral del día a día. Para ello, se debe replantear no solo el modelo educativo en el área de salud, sino que además se deben promover las competencias en los docentes como modelo profesional a sus estudiantes.

Un docente calificado que demuestra seguridad y altas competencias a sus estudiantes, al vivenciar cualidades tanto interpersonales como profesionales se constituye en un ejemplo de sensibilización y calidad de vida tanto en el área disciplinar, fomenta la motivación en los estudiantes, y representa un compromiso de mejora.

Se concluye que el proceso de prácticas pedagógicas representa un factor clave en los procesos de enseñanza en el área de salud, en cuanto a competencias docentes. Es importante apalancar la formación educativa a lo largo del desarrollo de la profesión, con un sistema innovador en las prácticas pedagógicas de los docentes del área.

\section{REFERENCIAS BIBLIOGRÁFICAS}

- Ángel M, Ruiz Py Rojas E. (2017). Propuesta de competencias profesionales para docentes de programas de salud en educación superior. Documento en línea disponible en: https:// revistas.unal.edu.co/index.php/revfacmed/article/view/58620/65125. Consulta: 24/10/18

- Basto, T. (2011). De las concepciones a las prácticas pedagógicas de un grupo de profesores universitarios. Documento en línea disponible en: https://revistas.javeriana. edu.co/index.php/MAGIS/article/view/3547. Consulta: 10/08/18

- Calderón, C. (2012). La descentralización educativa y el significado para el talento humano docente y directivo en la educación. Documento en línea disponible en: http:// ridum.umanizales.edu.co: 8080/xmlui/bitstream/handle/6789/830/Calder\%C3\%B3n_ Correa_Clara_Ines_2012.pdf? sequence=2). Consulta: 11/12/18

- Díaz Barriga, (2006). Estrategias docentes para el aprendizaje significativo. Documento en línea disponible en: http://formacion.sigeyucatan.gob.mx/formacion/materiales/4/4/d1/ p1/2.\%20estrategias-docentes-para-un-aprendizaje-significativo.pdf. Consulta:11/01/19

- Diaz A et al. (2018). Empathy in the Curriculum for Patient Care. Documento en línea disponible en: http://www.ccsenet.org/journal/index.php/gjhs/article/view/73488. Consulta:10/01/19

- Duque P, Rodríguez, J y Vallejo, S. (2013). Prácticas pedagógicas y su relación con el 
rendimiento académico. Documento en línea disponible en: http://biblioteca.clacso.edu. ar/Colombia/alianza-cinde-umz/20140805022434/paulaandreaduque. Consulta:10/01/19

- Medina E y Santeliz J. (2008). Estrategias de participación docente en una nueva propuesta de diseño curricular basada en competencias. Documento en línea disponible en: http:// www.scielo.org.ve/scielo.php?script=sci_arttext\&pid=S1316-49102008000400015. Consulta:29/09/18

- MEN (2009). Maestros competentes. Obtenido de maestros competentes. Documento en línea disponible en: https://www.mineducacion.gov.co/1621/article-195576.html. Consulta: $18 / 01 / 18$

- Perrenoud P.(2004). Desarrollar la práctica reflexiva en el oficio de enseñar: profesionalizacióny razónpedagógica. Documento enlínea disponibleen: https://coleccion. siaeducacion.org/sites/default/files/files/6_perrenoud_philippe_2007desarrollar_la_ practica_reflexiva.pdfMéxico D.F.: Graó; 2004. Consulta:11/07/18

- Restrepo, J y Navío, A. (2016). Las competencias del docente de maestría en universidades colombianas: apreciaciones de alumnos y profesores. Documento en línea disponible en: https://www.redalyc.org/pdf/1002/100250983010. Consulta:03/10/18

- Sistema nacional de información para la educación superior. (2017). Documento en línea disponible en: Estadísticas del ingreso estudiantil. Bogotá - Colombia: https:// www.mineducacion.gov.co/sistemasinfo/Informacion-a-la-mano/212400:Estadisticas. Consulta:23/11/18

- Tobón, T. (2013). Tobón, S. (2013). Formación integral y competencias. Pensamiento complejo, currículo, didáctica y evaluación (4ta. Ed.). Bogotá: ECOE. Documento en línea disponible en: https://www.researchgate.net/profile/Sergio_Tobon4/ publication/319310793_Formacion_integral_y_competencias_Pensamiento_complejo_ curriculo_didactica_y_evaluacion/links/59a2edd9a6fdcc1a315f565d/Formacionintegral-y-competencias-Pensamiento-complejo-curriculo-didactica-y-evaluacion.pdf. Consulta: 19/07/18

- Villardon L, Yániz C. (2006). Planificar desde competencias para promover el aprendizaje: el reto de la sociedad del conocimiento para el profesorado universitario. Documento en línea disponible en: https://dialnet.unirioja.es/servlet/libro?codigo=325795Bilbao. Consulta:08/09/18

- Zabalza, M. (2016). ser profesor universitario hoy. La cuestión universitaria. Consulta:18/01/19

- Zempoalteca, Barragan, Martínez y Guzmán. (2017). Formación en TIC y competencia digital en la docencia en instituciones públicas de educación superior. Documento en línea disponible en: http://www.scielo.org.mx/scielo.php?script=sci_arttext\&pid 
$=$ S1665-61802017000200080). Consulta:18/01/19

- Zúñiga, M y Jopia, B. (2007). La evaluación del desempeño docente en las universidades chilenas: diagnostico desde la perspectiva de las autoridades universitarias. Documento en línea disponible en: https://www.researchgate.net/publication/297517456_Evaluacion_ del_desempeno_docente_y_calidad_de_la_docencia_universitaria_2007-17Santiago de Chile, Chile. Cinda Mineduc. Consulta:06/09/18 